\title{
Evaluación de la intervención enseñanza: grupo en la consulta de enfermedad renal crónica avanzada
}

Accésit al XVII Premio de Investigación en Enfermería Nefrológica Janssen-Cilag

\author{
Josep Ma Gutiérrez Vilaplana - Elisabet Samsó Piñol - Judit Cosi Ponsa - Pilar Ibars i Moncasi - \\ Lourdes Craver Hospital
}

Hospital Universitari Arnau de Vilanova. LLeida

\section{RESUMEN}

En la etapa prediálisis, la calidad de vida del paciente con enfermedad renal crónica depende en gran medida del conocimiento que dispone de su enfermedad renal y del grado de adaptación a la misma. En nuestra consulta, los problemas abordados con más frecuencia en los pacientes con enfermedad renal crónica avanzada son: el afrontamiento inefectivo, el temor y conocimientos deficientes. El abordaje de estos problemas requiere muchas y variadas intervenciones. Conocedores de la importancia del papel que desempeñan los grupos, como sistemas sociales, en las conductas de salud y enfermedad, seleccionamos la intervención de la Clasificación de Intervenciones de Enfermería: 3S-5604 Enseñanza: grupo.

Los objetivos del estudio son: evaluar la intervención 3S-5604 enseñanza; grupo, en pacientes de la

\section{Correspondencia:}

Josep Ma Gutiérrez Vilaplana

Servicio de Nefrología

Hospital Universitari Arnau de Vilanova

Avda Rovira Roure N 80

25198 LLeida

e-mail:jgutierrez@arnau.scs.es consulta de enfermedad renal crónica avanzada, mediante resultados de enfermería.

El periodo del estudio va desde febrero del 2005 hasta abril del 2006. Las variables estudiadas: sexo, edad, nivel de estudios, presencia de acompañantes, resultados de enfermería antes y después de la intervención. Completaron la intervención un total de 24 pacientes de la Consulta de Insuficiencia Renal Crónica Avanzada seleccionados para dicha intervención.

La diferencia de puntuación para todos los resultados de enfermería, antes y después de la intervención, resultó significativa $p<0.05$.

La intervención 3S-5604 enseñanza: grupo mejoró en todos los resultados evaluados en los pacientes con enfermedad renal crónica en la consulta.

\section{PALABRAS CLAVE:}

- CONSULTA DE ENFERMEDAD RENAL CRÓNICA AVANZADA

- INTERVENCIÓN 3S-5604 ENSEÑANZA: GRUPO - RESULTADO DE ENFERMERÍA 
Evaluation of the teaching intervention: group in the advanced chronic kidney disease clinic

\section{Abstract}

In predialysis stage, Chronic Kidney Disease (CKD) patient quality of life depends on the knowledge he/she has of its renal disease and the adaptation level to the new CKD situation. In our CKD unit, renal patient problems more frequency faced are: Therapeutic regimen: individual, ineffective management, Knowledge deficit and Fear. The management of these problems requires many and varied interventions. Nurses know the group roles importance influence, as social systems, or health and disease behaviours. We selected nursing intervention: 3S-5604 Group Education.

The objective of the study is; to evaluate the nursing intervention 3S-56043S-5604 Group Education, in patients of the CKD unit, by means of nursing outcomes. Intervention study. The period of the study goes from February 2005 to April 2006. The studied variables: sex, age, studies degree, presence of companions, nursing outcomes before and after the intervention. The intervention was completed by a total of 24 patients of MRCA unit, selected for this intervention.

The difference of score for all the nursing outcomes before and after the intervention, was significant $p<0.05$. The intervention 3S-5604 3S-5604 Group Education improved in all the outcomes evaluated in the patients with chronic kidney disease in the CKD unit

\section{KEY WORDS}

- CHRONIC KIDNEY DISEASE UNIT

- INTERVENTION 3S-5604 GROUP: EDUCATION NURSING OUTCOME

\section{INTRODUCCIÓN}

La consulta de enfermedad renal crónica avanzada (ERCA) se crea en nuestro hospital en el año
1997, con el fin de abordar de forma multidisciplinar los diferentes aspectos biopsicosociales que presenta el paciente con ERC. Los pacientes tratados en la consulta ERCA corresponden principalmente a estadios 4 y 5 sin diálisis de la clasificación de enfermedad renal de las guías K/DOQI ${ }^{1}$.

En la etapa prediálisis, la calidad de vida del paciente con enfermedad renal crónica depende en gran medida del conocimiento que dispone de su enfermedad renal y del grado de adaptación a la misma.

La Organización Mundial de la Salud (OMS) a través de la Carta de Ottawa para la promoción de salud, define el fomento de los autocuidados como uno de los ejes principales, en la atención a la salud de las personas, incluyendo la potenciación de la autoevaluación y de la ayuda mutua².

En nuestra consulta, los problemas tratados con más frecuencia en los pacientes ERCA son:

Afrontamiento inefectivo: incapacidad para llevar a cabo una apreciación válida de los agentes estresantes para elegir adecuadamente las respuestas habituales o para usar los recursos disponibles.

Temor: respuesta a la percepción de una amenaza que se reconoce conscientemente como peligro.

Conocimientos deficientes: carencia o deficiencia de información cognitiva relacionada con un tema específico.

El abordaje de estos problemas requiere muchas y variadas intervenciones a lo largo de toda la etapa en que el paciente con ERC acude a la consulta. EI incremento del número de nuevos pacientes que precisan de nuestros cuidados, junto con el interés en la mejora en la calidad de los mismos, conducen al planteamiento de introducción de nuevas intervenciones.

Con esta intención de mejorar los cuidados se elige la intervención de Clasificación de Intervenciones de Enfermería (CIE): 3S-5604 Enseñanza: Grupo3. Definimos esta intervención como: el desarrollo, puesta en práctica y evaluación de un programa de educación para un grupo de personas con el mismo estado de salud. 
Entre las actividades, para llevar a cabo esta intervención, se seleccionan por consenso entre todo el equipo de la consulta las siguientes:

- Proporcionar un entorno que favorezca el aprendizaje

- Inclusión de la familia o ser querido

- Definir la población diana potencial

- Escribir los objetivos del programa

- Definir las áreas de contenido importante

- Hacer una lista de las estrategias de la enseñanza, los materiales educativos y las actividades de aprendizaje

- Proporcionar un programa escrito -que incluya fechas, horas y lugares de las sesiones/clases de enseñanza- para el personal y/o el paciente, si procede

- Controlar el tamaño y las competencias del grupo, si procede

- Orientar al paciente/ser querido al programa educativo y a los objetivos diseñados que hay que conseguir

- Adaptar los métodos/materiales educativos a las necesidades/características de enseñanza del grupo

- Evaluar el progreso del paciente en el programa y dominio del contenido

- Registrar el progreso del paciente en el registro médico permanente

- Evaluar el grado de consecución de objetivos del programa

- Remitir al paciente a otros especialistas/centros para conseguir los objetivos de enseñanza si precisa

La elección de una intervención para un paciente concreto forma parte de la toma de decisiones clínicas del profesional de enfermería, debiéndose tener en cuenta para ello factores como, los resultados deseados del paciente, las características del diagnóstico de enfermería, la base de investigación de la intervención, factibilidad de la ejecución, aceptabilidad para el paciente y la capacidad del profesional ${ }^{3}$.

La Clasificación de Resultados Enfermeros (CRE) nos proporciona un lenguaje estandarizado para los resultados que pueden medirse a lo largo de todo el proceso asistencial continuo, a la vez que aporta información esencial necesaria para que el resto del equipo multidisciplinar, consiga la continuidad y evaluación de los cuidados ${ }^{4}$.

Los resultados deseados en el paciente, especificados antes de elegir la presente intervención son:

- N-1302 Superación de problemas: Acciones para controlar los factores estresantes que ponen a prueba los recursos del individuo

- J-0906 Toma de decisiones: Capacidad para escoger entre dos o más alternativas

- 0-1404 Control del miedo: Acciones personales para eliminar o reducir los sentimientos incapacitantes de alarma, secundarios a una fuente identificable

- S-1803 Conocimiento del proceso de enfermedad: Grado de comprensión transmitido sobre el proceso de una enfermedad concreta

- S-1813 Conocimiento régimen terapéutico: Grado de comprensión transmitido sobre el régimen terapéutico específico

- S-1802 Conocimiento dieta: Grado de comprensión transmitido sobre la dieta

- S-1814 Conocimiento procedimiento terapéutico: Grado de comprensión transmitido sobre los procedimientos requeridos dentro de un régimen terapéutico

La importancia del papel que desempeñan los grupos, como sistemas sociales, en las conductas de salud y enfermedad les convierten en instrumentos fundamentales para la promoción, la prevención, el tratamiento, la recuperación y la intervención en los múltiples ámbitos de la salud psicosocial ${ }^{5}$.

El grupo se define como un conjunto de personas que tienen una finalidad y unos objetivos comunes, que interaccionan y que desempeñan diferentes roles, que tienen conciencia de que forman parte de un grupo y que establecen relaciones de interdependencia es decir que las acciones y respuestas de cada miembro pueden afectar e influir en los demás ${ }^{6}$. Los tipos de grupo más idóneos para nuestra intervención:

- Grupo Psicoeducativo: conducido por uno o dos profesionales, está indicado para trabajar en contextos socio-sanitarios, su finalidad es enseñar hábitos saludables básicos y necesarios en 
determinadas patologías crónicas. Promueven cambios de actitudes hacia la salud.

- Grupo de apoyo: Compuesto por personas que comparten algún tipo de problema que altera o modifica aspectos de su normal funcionamiento como por ejemplo situaciones crónicas (diabéticos, programas de diálisis...). La pertenencia a este grupo proporciona nuevos lazos y relaciones sociales a sus miembros. Fomenta directamente la salud y la reducción del malestar psicológico ya que permiten al individuo hacer una mejor valoración de su propia situación. El grupo ofrece recursos de afrontamiento para paliar emociones negativas, proporciona mayor control sobre la situación, aumento de la autoestima y un estado de ánimo positivo. También brinda una oportunidad de interacción social regularizada con recepción de feedback de los demás miembros ayudando a identificar síntomas y actuar con rapidez ante cualquier desajuste ${ }^{7}$.

\section{Objetivo}

Evaluar la intervención 3S-5604 Enseñanza: grupo, en pacientes de la consulta de enfermedad renal crónica avanzada, mediante resultados de enfermería.

\section{Material y método}

Pacientes. Se incluyeron los pacientes de la consulta ERCA de nuestro hospital, seleccionados para la intervención 3S-5604 Enseñanza: grupo. Todos estos pacientes habían sido informados previamente sobre la intervención y habían dado su consentimiento, tras la intervención 3S-5606. Enseñanza: individual.

Dos pacientes fueron excluidos del estudio: abandono debido a la no aceptación de la intervención, y por no cumplimentar la totalidad de las sesiones refiriendo problemas laborales. Completaron la intervención un total de 24 pacientes.

Se formaron 4 grupos de pacientes del tipo: grupo psicoeducativo y de apoyo ${ }^{7}$, con un número de 6-7 pacientes más un acompañante por paciente. La in- tervención se estructuró en 8 sesiones de dos horas cada una, durante 6 meses. El periodo del estudio va desde febrero del 2005 hasta abril del 2006. Los diferentes temas seleccionados fueron:

- El riñón y su funcionamiento

- Tratamiento sustitutivo renal en pacientes con enfermedad renal crónica avanzada: hemodiálisis, diálisis peritoneal y trasplante renal

- Aspectos legales

- Minusvalías. Aspectos sociales

- Medidas higiénico-dietéticas

- Riesgo vascular

o Trasplante renal

Estos temas se abordaron mediante presentaciones en Power Point, talleres didácticos y coloquios entre los participantes. Las charlas fueron impartidas por el personal sanitario de la consulta y por pacientes voluntarios ya en tratamiento renal sustitutivo. Se proporcionó en cada charla material didáctico impreso con el contenido de las mismas, dicho material fue elaborado por el equipo de la consulta ERCA.

Diseño. Se realizó un estudio de intervención. Las variables estudiadas: sexo, edad, nivel de estudios, presencia de acompañantes, resultados de enfermería antes y después de la intervención, fueron recogidas mediante entrevista personal, y registro de la historia clínica. Se realizó una estadística descriptiva de las variables basales y un estudio de muestras pareadas para evaluar el efecto de la intervención sobre los 15 diferentes indicadores de resultado, valorándose la significación para una $p<0.05$. En aquellos resultados de enfermería que para su evaluación se precisó más de un indicador de resultados, se utilizó la media de puntuación de los mismos. Los indicadores de resultado analizados fueron los siguientes:

- 130204 Refiere disminución de estrés

- 130206 Busca información sobre la enfermedad y su tratamiento

- 130207 Modifica el estilo de vida cuando se requiere

- 130217 Refiere disminución de los sentimientos negativos 
- 090608 Compara alternativas

- 090609 Escoge entre varias alternativas

- 140403 Busca información para reducir el miedo

- 140417 Controla la respuesta del miedo

- 180302 Descripción del proceso de la enfermedad

- 181304 Descripción de los efectos esperados del tratamiento

- 181310 Descripción de los procedimientos prescritos

- 180207 Descripción de las comidas que deben evitarse

- 180210 Selección de comidas recomendadas por la dieta

- 180212 Desarrollo de estrategias para cambiar hábitos alimentarios

- 181401 Descripción del procedimiento terapéutico

Los indicadores de resultado seleccionados, fueron medidos según escala específica (del 1 al 5) y registrados por enfermería antes de la primera reunión (antes) y en la visita posterior a la última reunión (después).

EI análisis estadístico se realizó mediante SPSS 11.5 .

\section{RESULTADOS}

\section{Demográficos}

La edad media de la población de la consulta: $64,5 \pm 11,26$ años (40-84). La distribución por sexo: $79,2 \%$ varones $(n=19)$ y $20,8 \%$ de mujeres $(n=5)$. Presencia de acompañantes en la intervención 3S5604 Enseñanza: grupo. El 62,5\% ( $n=15)$ de los pacientes acudieron acompañados frente al 37,5\% $(n=9)$ que acudieron solos. El $68,4 \%$ de los hombres $(n=13)$ y el $40 \%$ de las mujeres $(n=2)$ asistieron acompañados a las reuniones.

El nivel de estudios de los pacientes se refleja en la figura 1.

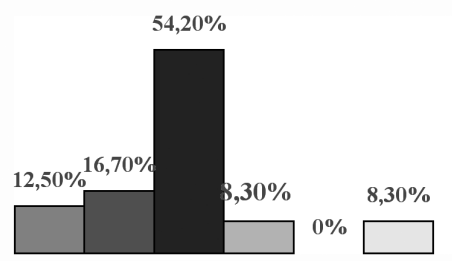

$\square$ Estudios 1os incompletos

口 Estudios 1os completos

- Graduado escolar

$\square$ BUP/FP

$\square$ Universitarios grado medio

$\square$ Universitario grado superior

Figura 1. Nivel de estudios de los pacientes
La evaluación de los diferentes resultados de enfermería se pueden observar en las figuras 2 y 3 .
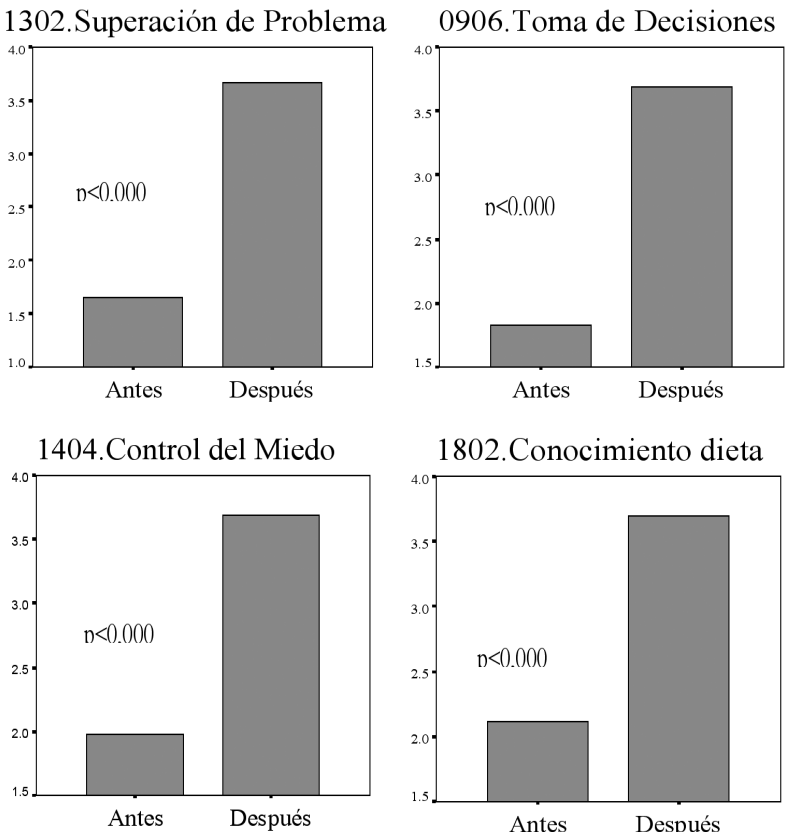

1802. Conocimiento dieta

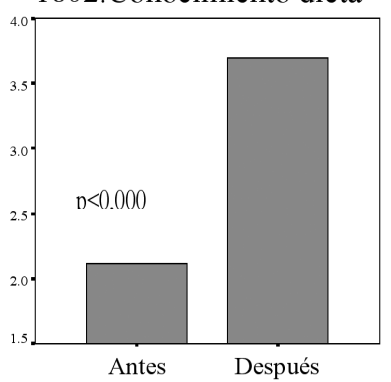

Figura 2. Evaluación de los resultados

1803. Conocimiento del

1813.Conocimiento

Proceso de Enfermedad

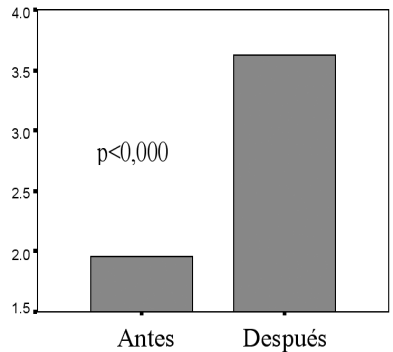

regimen terapéutico

1814. Conocimiento
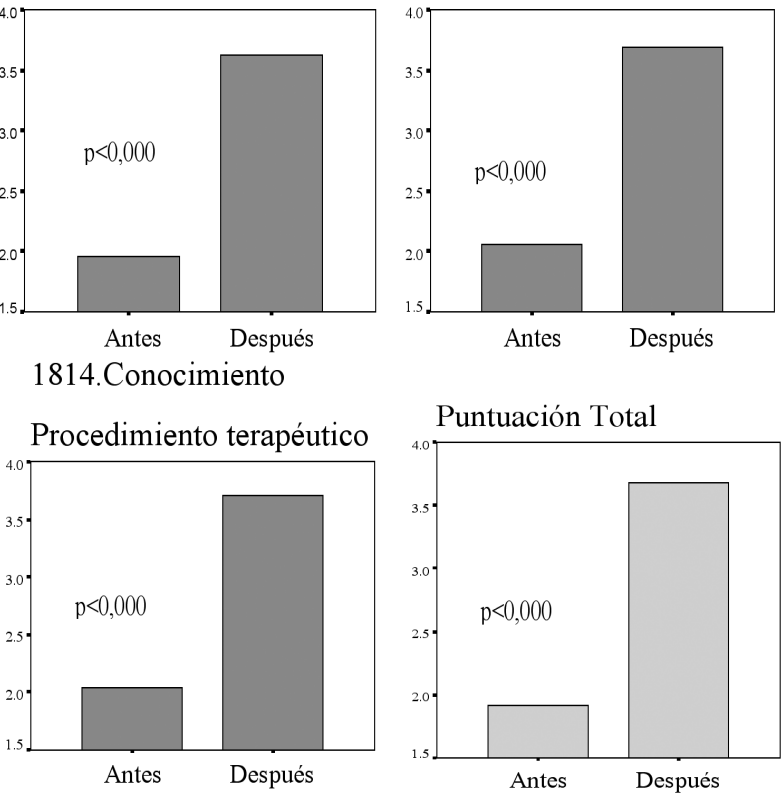

Puntuación Total

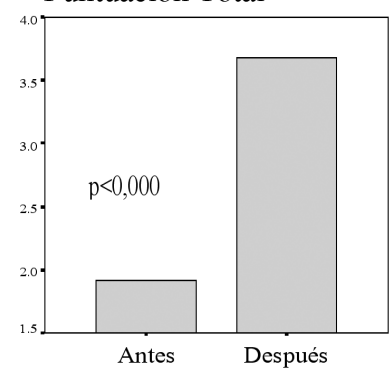

Figura 3. Evaluación de los resultados

\section{Discusión}

Mediante la intervención 3S-5604 Enseñanza: grupo pudimos incidir en tres de los diagnósticos enferme- 
ros, que tras evaluación inicial de enfermería, detectamos en la mayoría de pacientes con enfermedad renal crónica avanzada de la consulta ERCA. Esta intervención facilita el abordaje de aspectos como el temor, la educación, y la elección de técnica de tratamiento renal sustitutivo.

La intervención 3S-5604 Enseñanza: grupo mejoró tanto a nivel individual como en la totalidad del grupo, cada uno de los diferentes resultados de enfermería valorados.

Esta intervención incrementa los cuidados recibidos por el paciente en la consulta ERCA, fomentando una relación terapéutica entre los pacientes con enfermedad renal crónica, sus acompañantes y el equipo multidisciplinar.

El resultado de la presente evaluación se corresponde con la impresión subjetiva que el equipo sanitario ha percibido a lo largo del tiempo de seguimiento de la intervención.

La mejoría de los resultados en estos pacientes resultó ser un buen criterio para la evaluación de innovaciones clínicas como es el caso de una nueva intervención. Por tanto creemos que la intervención 3S-5604 Enseñanza: grupo mejora significativamente en todos los resultados de enfermería evaluados en los pacientes con enfermedad renal crónica en la consulta de ERCA.

\section{Agradecimientos}

Agradecemos la colaboración de todos los miembros de este nuevo equipo multidisciplinario de salud; pacientes, acompañantes, enfermeras y médicos, sin su colaboración esta magnífica experiencia no hubiera sido posible.

\section{Bibliografía}

1. K/DOQI Clinical Practise guidelines for Chronic Kidney Disease: Evaluation, Classification and Stratification. Am J Kidney Dis. 2003; 39 (Suppl1): S1-S89.

2. The Ottawa Charter for Health Promotion. WHO Reg Publ Eur Ser. 1992; 44: 1-7.

3. McCloskey J, Bulechek G. Clasificación de intervenciones de Enfermería (CIE). Ed. Madrid: Harcourt Mosby; 2002.

4. Bulechek GM. Nursing interventions: effective nursing treatments. Philadelphia; Ed. Saunders; 2000.

5. Johnson M, Maas M, Moorhead S. Clasificación de resultados de Enfermería (CIE). Madrid: Ed. Harcourt Mosby; 2002.

6. Rodríguez-Marín J. Psicología social de la salud. Madrid: Síntesis; 1995.

7. Pérez RM, Sáez S. El grupo en la promoción y la educación para la salud. Lleida: Ed. Milenio; 2004.

8. Barrón A. Modelos teóricos del apoyo social. SeviIla: Ed Eudema; 1993. 